\title{
Resolving the challenges of time and distance
}

\author{
Filippo Lanubile • Rafael Prikladnicki • Erran Carmel • \\ Rini van Solingen
}

Published online: 5 April 2014

(C) Springer Science+Business Media New York 2014

Software and IT industries are today truly global and so is Software Engineering. High-tech companies are now located in more than 100 countries, making Global Software Engineering (GSE) a fact of life. The Internet has facilitated a massive dispersion of high-tech work and has completely changed how we humans deal with time and distance. However, the dispersion over time and space require novel techniques, tools, and practices from many disciplines to overcome challenges and to take advantage of all the opportunities that global software engineering offers. Developing software has become a global phenomenon with both successes and failures. At the same time, the software industry has been quick to take advantage of all the opportunities that global software engineering offers but it is still learning to overcome the challenges that distance brings in.

This special section on Global Software Engineering consists of two papers that present innovative ideas on not only how to resolve the challenges of time and distance, but also on how to cope with the growing complexity of effectively managing GSE work.

The first paper is "Agile vs. Structured Distributed Software Development: A Case Study" (DOI: 10.1007/s10664-013-9271-y). In their work, the authors explore a central question in global software development: does the choice of process affect the success of a distributed development project? Based on a mix of quantitative and qualitative analysis of data related to 66 distributed development projects in three continents, the main finding is the lack of a significant difference between project outcomes and the type of development process, agile or structured.

F. Lanubile $(\bowtie)$

Dipartimento di Informatica, University of Bari, Bari, Italy

e-mail: filippo.lanubile@uniba.it

R. Prikladnicki

Pontifícia Universidade Católica do Rio Grande do Sul (PUCRS), Porto Alegre, RS, Brazil

e-mail: rafael.prikladnicki@pucrs.br

E. Carmel

Kogod School of Business, American University, Washington, DC, USA

e-mail: carmel@american.edu

R. van Solingen

Department of Electrotechnics, Mathematics and Computer Science, Delft University of Technology, Delft, The Netherlands

e-mail: D.M.vanSolingen@tudelft.nl 
The second paper is entitled "From offshore outsourcing to insourcing and partnerships: four failed outsourcing attempts" (10.1007/s10664-013-9272-x), where the authors analyze failure and recovery patterns of four small-medium software companies. Based on the qualitative analysis of data collected from multiple sources, the study identifies the main challenges of global software development and highlights the switch from offshore outsourcing to offshore insourcing and partnerships.

These two papers are concrete examples of high-quality empirical work in the field of global software engineering - a field which has a need for deeper research. Both papers are extended papers from the IEEE Conference series on Global Software Engineering (icgse.org), a community that shares industrial and academic results and practices on how to really comprehend and manage Global Software Engineering. 